\title{
Intracoronary bone marrow cell application for terminal heart failure in children
}

\author{
Stefan Rupp, ${ }^{1}$ Christian Jux, ${ }^{1}$ Halvard Bönig, ${ }^{2}$ Jürgen Bauer, ${ }^{1}$ Torsten Tonn, ${ }^{2, *}$ Erhard Seifried, ${ }^{2}$ \\ Stefanie Dimmeler, ${ }^{3}$ Andreas M. Zeiher, ${ }^{3}$ Dietmar Schranz ${ }^{1}$ \\ ${ }^{1}$ Pediatric Heart Center, Justus-Liebig University Giessen, Giessen; ${ }^{2}$ German Red Cross Blood Service \\ Baden-Württemberg-Hessen and Institute for Transfusion Medicine and Immunohaematology, Goethe University \\ Frankfurt, Frankfurt, Germany; ${ }^{3}$ Department of Cardiology, Goethe University Frankfurt, Frankfurt, Germany
}

\begin{abstract}
Introduction: In spite of tremendous progress in the medical and surgical treatment of children with congenital heart disease and dilated cardiomyopathy achieved during the past few decades, for some children a heart transplant remains the only option. Clinically relevant benefits of intracoronary injection of autologous stem cells on cardiac function and remodelling have been demonstrated in adult patients with acute myocardial infarction. Experience with autologous stem cell therapy in children with severe congenital or acquired pump failure is limited to a small number of case reports. Method and results: Between 2006 and 2010, nine severely ill children were treated with intracoronary infusion of autologous bone marrow-derived mononuclear cells as part of a compassionate therapy in our centre. No procedure-related unexpected adverse events occurred. There was one patient on extracorporeal membrane oxygenation who died of haemorrhage unrelated to the procedure; three patients proceeded to heart transplantation once a donor heart became available. The other five patients showed an improvement with respect to New York Heart Association classification (greater than or equal to 1), brain natriuretic peptide serum levels, and ejection fraction. Conclusion: Similar to adults, intracoronary injection of autologous bone marrow cell is technically feasible and safe for children. On the basis of our data, we propose to perform a pilot study for children with congestive heart failure, to formally assess the efficacy of intracoronary autologous bone marrow cell therapy.
\end{abstract}

Keywords: Cardiac regeneration; heart insufficiency; dilated cardiomyopathy

Received: 31 July 2011; Accepted: 20 December 2011; First published online: 13 February 2012

A MONG THE MORE SEVERE CONGENITAL ABNORMALities, cardiac malformations play an important qualitative and quantitative role. The estimated incidence of 10 per 1000 live births seems to be a conservative estimate for the prevalence of cardiac malformations. ${ }^{1}$ Owing to tremendous advances in the medical and surgical care of children with congenital heart disease over the past few decades, survival into adulthood has become a reality; however, some

Correspondence to: Professor Dr D. Schranz, MD, Pediatric Heart Center, Justus-Liebig University, Feulgenstrasse 12, 35385 Giessen, Germany. Tel: 049 641 9943461; Fax: 049641 9943469; E-mail: Dietmar.Schranz@paediat.med. uni-giessen.de

*Institute for Transfusion Medicine, German Red Cross Blood Donor Service East, Medical Faculty Carl Gustav Carus, TU Dresden, Dresden, Germany. patients with complex malformations, despite best surgical and medical efforts, ultimately require a heart transplant.

Dilated cardiomyopathy is also associated with considerable morbidity and mortality. About one-third of children diagnosed with dilated cardiomyopathy die within the first year after diagnosis, unless they receive a heart transplant. ${ }^{2-4}$

The Pediatric Heart Centre in Giessen offers the full therapeutic spectrum for heart failure in children, ranging from basic anticongestive treatment to implantation of assist devices and heart transplantation. Because of side effects of the immunosuppressive agents and the decreasing function of the transplanted organ over time, the long-term outcome 
after heart transplantation remains limited. The modest long-term outcome and the paucity of donor organs $^{5}$ fuel the ongoing search for improved medical and surgical therapy, and for therapeutic alternatives in patients with congenital heart disease and dilated cardiomyopathy.

Over the last decade, it has become apparent that the heart possesses modest regenerative capacity with respect to cardiomyocytes and new blood vessel formation. ${ }^{6}$ Human bone marrow-derived proangiogenetic cells were shown to improve angiogenesis and neovascularisation. ${ }^{7}$ Moreover, a sizeable fraction of cardiomyocytes refreshes the heart after injury or under conditions of pressure overload. ${ }^{8,9}$ However, in chronic pathological conditions of cardiac de-compensation, the endogenous repair capacity may not be sufficient. Recently, it was shown that in healthy individuals cardiomyocyte renewal decreases from $2 \%$ turnover annually at the age of 10 years to $1 \%$ at the age of 27 years and $0.45 \%$ at the age of 75 years. ${ }^{10}$ Extrapolation of this scale to children younger than 10 years suggests an even higher amount of cardiomyocyte renewal in healthy infants. Children with anomalous left coronary arteries arising from the pulmonary artery, who present with severely reduced ventricular function, generally recover after surgery and acquire normal ventricular function over time. ${ }^{\$ 1}$

Initial pilot studies with intracoronary infusion of autologous bone marrow-derived cells in adults with acute myocardial infarction reported an improvement in global left ventricular ejection fraction, reduced end-systolic left ventricular volumes, and improved perfusion in the infarcted area. ${ }^{12-15}$ These beneficial effects were confirmed in the thus far largest double-blind, randomised multi-centre REPAIR-AMI trial. ${ }^{16}$ However, these data are not uncontested, as another large study did not show any benefit on left ventricular functional parameters. ${ }^{17}$ Subtle differences in cell processing, affecting cell functionality, were proposed as an explanation for the divergent outcomes. Meta-analyses confirmed clinically relevant benefits of intracoronary bone marrow cell therapy on cardiac function and remodelling in adult patients. ${ }^{18,19}$

We hypothesise that particularly in young or even very young patients considerable regenerative potential of the heart is preserved and that cardiac cell therapy might be of even greater value than in adults.

\section{Methods}

Between 2006 and 2010, we performed intracoronary infusion of autologous bone marrow-derived mononuclear cells in nine patients at our heart transplantation centre. In each of the described cases, congestive heart failure developed despite maximum surgical and medical care. At the time of cell therapy, three patients were on assist devices. Positive inotropic substances were used in four patients and a careful switch to anticongestive treatment was performed in two patients. Anticongestive treatment consisted of beta-1-selective beta-blockers, angiotensin converting enzyme-inhibitors, and spironolactone. Anticongestive treatment proved not to be sufficient, because both patients additionally required diuretics. In all, five patients were listed for heart transplantation; in one case, heart transplantation was not an option because the patient was from a nonEuropean Union country.

In all cases, intracoronary bone marrow cell administration was considered as compassionate treatment to stabilise the patients' clinical condition. Ejection fraction, brain natriuretic peptide serum levels, and New York Heart Association classification were regularly assessed during followup according to the clinical conditions of the patient. The local ethics committee approved the procedure as a compassionate approach, and written informed consent was obtained from the parents.

In each case, a bone marrow aspirate of 1-2 millilitres per kilogram - maximum of 60 millilitres - was drawn under systemic sedation and analgesia, as well as local anaesthesia. Bone marrow and autologous serum was processed according to Good Manufacturing Practice standards ${ }^{13}$ by the Institute for Transfusion Medicine and Immunohematology of the Goethe University Clinic, Frankfurt. Cells were suspended in a 4-12-millilitre solution and re-infused on the same day using the stop-flow technique as described before. ${ }^{13}$

Over-the-wire coronary balloon catheters of widths between 1.5 and 2.5 millimetres and lengths of 8 to 20 millimetres were advanced into the selected coronary arteries without the use of an additional guiding catheter. Coronary arteries were selected on the basis of regions of pronounced dysfunction of the supplied ventricular area (Fig 1a). Infused volumes and selected arteries are shown in Table 2. To allow for adhesion and potential transmigration of the infused cells through the endothelium, the balloon was inflated with low pressure (less than 1-2 atmospheres) to completely block blood flow for 3 minutes, while the cell suspension was infused distally to the occluding balloon through the central port of the balloon catheter, after the guide wire was removed (Fig 1b). After completion of intracoronary cell administration, coronary angiography was repeated to ascertain vessel patency and unimpeded flow of contrast material.

Statistical assessment of the possible benefits of cardiac cell therapy is beyond the scope of this 

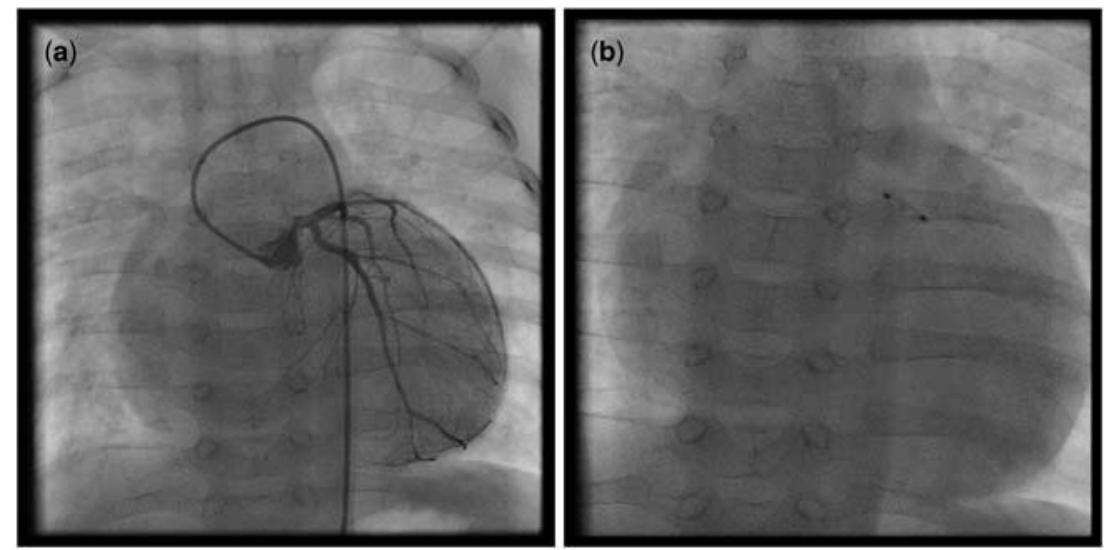

Figure 1.

Infusion of bone marrow cells (BMC).

manuscript. Owing to the fact that all patients were severely ill, cell therapy was only part of the strategy and heart transplantation remained the therapeutic goal, provided that a suitable donor organ would become available before a significant improvement in clinical condition was seen (New York Heart Association improvement greater than or equal to 1 for at least 3 months).

\section{Results}

In all, nine patients received intracoronary infusion of bone marrow cells as described. During application using the stop-flow technique, moderate ST-segment changes were documented; however, no increase in cardiac troponin $\mathrm{T}$ was observed after the procedure, providing evidence that intracoronary application of autologous bone marrow-derived cells is feasible and safe. The specification of the infused cells is given in Table 1. No acute adverse events were observed; all treated vessels were and remained patent and unaffected. The characteristics of the patients are summarised in Table 2. After donor organs became available, two patients proceeded to heart transplantation - 48 days and 32 days, respectively, after cell therapy, patient I and patient II that is, before the efficacy of cell therapy could be ascertained.

The first patient suffered from a dilated cardiomyopathy likely because of an anthracyclin that she had received for treatment of nephroblastoma at the age of 5 years. She had been on a left ventricular assist device at the time of cell therapy and proceeded to heart transplantation after 48 days.

The second patient with idiopathic dilated cardiomyopathy was treated with a maze operation for the right atrium and a Carpentier-Edwards Ring at the tricuspid valve 2 months before the cell
Table 1. Characteristics of infused cells.

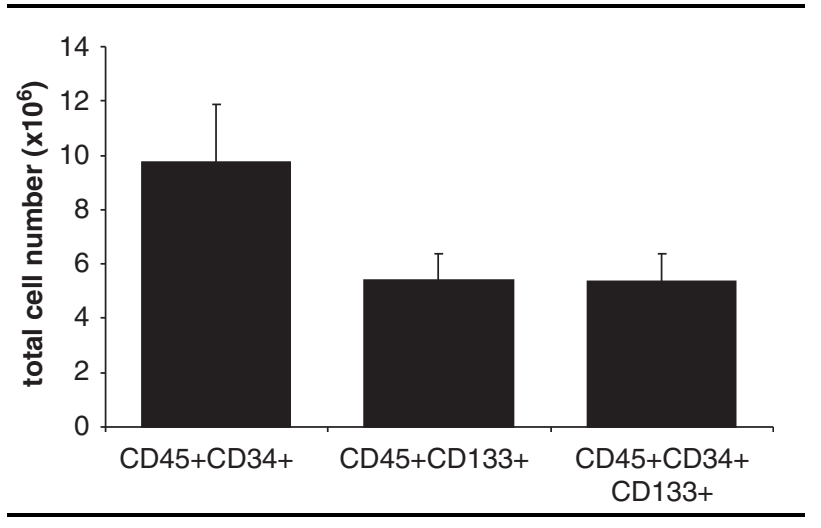

therapy. Further deterioration of the clinical condition led to listing for heart transplantation and a cell therapy was performed. A donor heart became available 32 days after cell therapy, and the patient received a heart transplant before the efficacy of cell therapy could be ascertained.

The third patient with idiopathic dilated cardiomyopathy who had been on an assist device showed a clinical benefit after cell therapy, which cannot be clearly discriminated from beneficial effects from the assist device that was implanted 4 weeks before cell therapy. The improvement of his clinical condition allowed removal of the assist device. However, another clinical deterioration followed and a secondary assist device was implanted 7 months after explantation of the first device. The patient was finally transplanted when an organ became available.

The fourth patient suffered from double outlet right ventricle, that is, Taussig-Bing Complex. This patient was postnatally treated with a ductal stent and a pulmonary artery banding. After surgical repair at the age of 5 months - arterial 


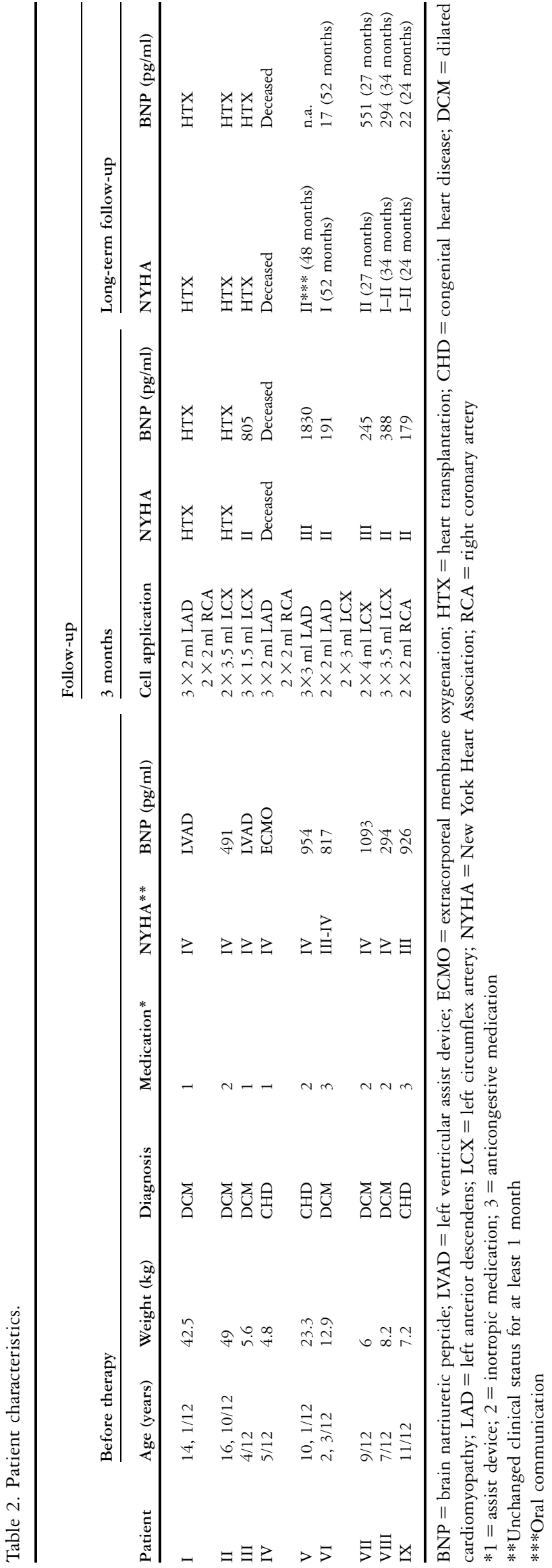

switch operation with occlusion of the ventricular septal defect and aortic arch repair - the patient showed a severely reduced cardiac and pulmonary function and was treated with extracorporeal membrane oxygenation. An autologous bone marrow cell therapy was performed, yet the patient died 5 weeks later while on extracorporeal membrane oxygenation with severely reduced cardiac and pulmonary function. She succumbed to a haemorrhagic infarction of the middle cerebral artery and pulmonary haemorrhage.

The fifth patient with pulmonary atresia and ventricular septal defect suffered from left ventricular dysfunction due to coronary ischaemia thrombus formation in the left anterior coronary descending from the right coronary artery - after surgical repair - including unifocalisation of main pulmonary collateral arteries, homograft implantation at the side of pulmonary atresia, and closure of the atrial septal defect and ventricular septal defect.

The sixth patient was already reported in 2008 as a case report. ${ }^{20} \mathrm{He}$ suffered from dilative cardiomyopathy caused by a non-acute myocarditis.

The seventh patient suffered from severe dilated left ventricular cardiomyopathy from birth, probably due to ischaemia presumed secondary to opioid abuse of the mother during pregnancy.

The eighth patient suffered from dilative cardiomyopathy with severe mitral insufficiency caused by perinatal Coxsackie B Virus myocarditis and an infarction in the region of the left circumflex coronary artery. Late enhancement was documented in the infarcted area with magnetic resonance imaging, and autologous bone marrow cell therapy was performed several weeks after the ischaemic event. After 3 weeks, the mitral valve was reconstructed with a bioabsorbable Kalangos ring and fixation of the posterior leaflet. After 1 week of the operation, the patient developed ventricular and supraventricular tachycardia requiring amiodarone treatment. The clinical condition of the patient significantly improved in the following months, no arrythmia was documented in the follow-up, and amiodarone was discontinued 5 months later.

The ninth patient - age 11 months - had a hypoplastic left heart syndrome after hybrid comprehensive stage two, already reported before. ${ }^{21}$ In response to the cell therapy, his condition improved significantly. Meanwhile, total cava-pulmonary connection as stage III is completed.

\section{Discussion}

Here we report that autologous cardiac cell administration is technically feasible and safe in children. Overall, no procedure-related unexpected adverse events occurred in the present series. 
The severity of the initial clinical status of the patients limits a statistical assessment of the possible benefits of intracoronary administration of bone marrow-derived cells. However, in the absence of good evidence for the value of intracoronary bone marrow cell administration in paediatric heart disease, denial of an available transplant would have been unethical. Therefore, according to our protocol, patients received heart transplants after cell therapy, as a donor organ became available. However, one patient died soon after cell therapy of infarction of the middle cerebral artery and lung haemorrhage while being on extracorporeal membrane oxygenation. Both events were not temporally related to the cell therapy, and thus likely causally unrelated.

There were three patients with dilated cardiomyopathy who showed a definitive improvement in their clinical condition, brain natriuretic peptide serum levels, and ejection fraction during shortterm (2-3 months) and long-term (24-52 months) follow-up. There were two further patients with congenital heart disease who also apparently benefited from autologous intracoronary bone marrow-derived cell application. The temporal association of cell therapy and clinical improvement suggests a causal relationship, although this observation cannot formally be ascertained.

The beneficial effect of intracoronary administration of bone marrow-derived cells on neovascularisation is conclusively documented. ${ }^{7}$ However, the process of cardiomyocyte renewal, which remains controversially discussed in adult mammals, might play a much more prominent role in children, as cardiomyocyte turnover in children is higher than in adults. ${ }^{10}$ In addition, direct clinical evidence for an enhanced regenerative capacity of the paediatric myocardium $^{11}$ has been provided. In comparison with adults and patients with risk factors for cardiovascular diseases, the children's bone marrow showed considerably higher content of cells expressing the stem cell markers CD34 and CD133, which may reflect a superior regenerative capacity of juvenile bone marrow.

These encouraging data may indeed serve as a rationale to perform a pilot study in children with refractory congestive heart failure, in order to assess possible beneficial effects of autologous intracoronary bone marrow cell therapy. However, it should be noticed that dilated cardiomyopathy subsumes patients with one or two dilated ventricles with a variety of underlying (heart) diseases. In addition, patients with genetic defects may present with the diagnosis of dilated cardiomyopathy. Especially in these patients, autologous cell therapy might not be beneficial, because genetic defects in cardiomyogenesis might be responsible for the clinical syndrome; that is, in these patients, cardiac regeneration might be genetically limited. Owing to the variety of underlying nosologic entities, a controlled study with patients suffering from any dilated cardiomyopathy might not be performed in first line. Instead, we are planning a pilot study for infants and children with single ventricular hearts, Norwood stage II and III, reduced heart function, and severely impaired clinical status - New York Heart Association III), in order to ascertain a potential beneficial effect of intracoronary infusion of autologous bone marrow-derived mononuclear cells in a rather homogeneous group of patients with presumed cardiac regenerative capacity.

\section{References}

1. Pierpont ME, Basson CT, Benson DW Jr, et al. Genetic basis for congenital heart defects: current knowledge: a scientific statement from the American Heart Association Congenital Cardiac Defects Committee, Council on Cardiovascular Disease in the Young: endorsed by the American Academy of Pediatrics. Circulation 2007; 115: 3015-3038.

2. Arola A, Tuominen J, Ruuskanen O, Jokinen E. Idiopathic dilated cardiomyopathy in children: prognostic indicators and outcome. Pediatrics 1998; 101: 369-376.

3. Nugent AW, Daubeney PE, Chondros P, et al. The epidemiology of childhood cardiomyopathy in Australia. N Engl J Med 2003; 348: 1639-1646.

4. Alvarez J, Wilkinson JD, Lipshultz SE, for the Pediatric Cardiomyopathy Registry Study Group. Outcome predictors for pediatric dilated cardiomyopathy: a systematic review. Progress in Pediatric Cardiology 2007; 23: 25-32.

5. Almond CS, Thiagarajan RR, Piercey GE, et al. Waiting list mortality among children listed for heart transplantation in the United States. Circulation 2009; 119: 717-727.

6. Anversa P, Leri A, Rota M, et al. Concise review: stem cells, myocardial regeneration, and methodological artifacts. Stem Cells 2007; 25: 589-601.

7. Urbich C, Dimmeler S. Endothelial progenitor cells: characterization and role in vascular biology. Circ Res 2004; 95: 343-353.

8. Hsieh PC, Segers VF, Davis ME, et al. Evidence from a genetic fate-mapping study that stem cells refresh adult mammalian cardiomyocytes after injury. Nat Med 2007; 13: 970-974.

9. Rupp S, Koyanagi M, Iwasaki M, et al. Characterization of long-term endogenous cardiac repair in children after heart transplantation. Eur Heart J 2008; 29: 1867-1872.

10. Bergmann O, Bhardwaj RD, Bernard S, et al. Evidence for cardiomyocyte renewal in humans. Science 2009; 324: 98-102.

11. Lange R, Vogt M, Horer J, et al. Long-term results of repair of anomalous origin of the left coronary artery from the pulmonary artery. Ann Thorac Surg 2007; 83: 1463-1471.

12. Strauer BE, Brehm M, Zeus T, et al. Repair of infarcted myocardium by autologous intracoronary mononuclear bone marrow cell transplantation in humans. Circulation 2002; 106 : 1913-1918.

13. Assmus B, Schachinger V, Teupe C, et al. Transplantation of progenitor cells and regeneration enhancement in acute myocardial infarction (TOPCARE-AMI). Circulation 2002; 106: 3009-3017.

14. Fernandez-Aviles F, San Roman JA, Garcia-Frade J, et al. Experimental and clinical regenerative capability of human bone marrow cells after myocardial infarction. Circ Res 2004; 95: 742-748. 
15. Wollert KC, Meyer GP, Lotz J, et al. Intracoronary autologous bone-marrow cell transfer after myocardial infarction: the BOOST randomised controlled clinical trial. Lancet 2004; 364: 141-148.

16. Schachinger V, Erbs S, Elsasser A, et al. Intracoronary bone marrow-derived progenitor cells in acute myocardial infarction. N Engl J Med 2006; 355: 1210-1221.

17. Lunde K, Solheim S, Aakhus S, et al. Intracoronary injection of mononuclear bone marrow cells in acute myocardial infarction. N Engl J Med 2006; 355: 1199-1209.

18. Abdel-Latif A, Bolli R, Tleyjeh IM, et al. Adult bone marrowderived cells for cardiac repair: a systematic review and metaanalysis. Arch Intern Med 2007; 167: 989-997.
19. Lipinski MJ, Biondi-Zoccai GG, Abbate A, et al. Impact of intracoronary cell therapy on left ventricular function in the setting of acute myocardial infarction: a collaborative systematic review and meta-analysis of controlled clinical trials. J Am Coll Cardiol 2007; 50: 1761-1767.

20. Rupp S, Bauer J, Tonn T, et al. Intracoronary administration of autologous bone marrow-derived progenitor cells in a critically ill two-yr-old child with dilated cardiomyopathy. Pediatr Transplant 2009; 13: 620-623.

21. Rupp S, Zeiher AM, Dimmeler S, et al. A regenerative strategy for heart failure in hypoplastic left heart syndrome: intracoronary administration of autologous bone marrow-derived progenitor cells. J Heart Lung Transplant 2010; 29: 574-577. 